\title{
Narrow linewidth Terahertz Signal Generation using a Dual-Mode Semiconductor Fabry-Pérot Laser and a Uni-travelling Carrier Photodiode
}

\author{
Guillaume Ducournau ${ }^{\mathrm{a}}$, Alexandre Beck ${ }^{\mathrm{a}}$, Emilien Peytavit ${ }^{\mathrm{a}}$, Tahsin Akalin ${ }^{\mathrm{a}}$, Jean-François Lampin ${ }^{\mathrm{a}}$, \\ Sylwester Latkowski ${ }^{\mathrm{b}}$, Josué Parra-Cetina ${ }^{\mathrm{b}}$, Ramón Maldonado-Basilio ${ }^{\mathrm{b}}$ and Pascal Landais ${ }^{\mathrm{b}}$ \\ a Institut d'Electronique de Microélectronique et de Nanotechnologie, Université de Lille 1, UMR \\ CNRS 8520, Avenue Poincaré B.P. 60069, F-59652 Villeneuve d'Ascq Cédex, France \\ ${ }^{b}$ Research Institute for Networks and Communications Engineering, Dublin City University, Glasnevin, \\ Dublin 9, Ireland
}

\begin{abstract}
A narrowband terahertz signal generated by a uni-travelling carrier photodiode (UTC-PD) interfaced with a dual-mode Fabry-Pérot laser diode is demonstrated. A beat tone corresponding to the free spectral range is generated on the UTC-PD, and radiated by a transverse-electromagnetic-horn antenna. All components involved in this experiment operate at room temperature. The terahertz signal at a frequency of 372 GHz is recorded, and the linewidth is $17 \mathrm{MHz}$. The linewidth and the tunability of the emitted terahertz wave are analyzed, as a function of the laser bias.
\end{abstract}

\section{INTRODUCTION AND BACKGROUND}

$\mathrm{S}_{\mathrm{b}}$ EMICONDUCTOR lasers can produce a terahertz emission either using direct or indirect schemes [1-4]. In the direct case, a spectrum tailored Fabry-Pérot (FP) laser diode, designed for $370 \mathrm{GHz}$ mode separation, dc-biased at room temperature, has demonstrated its potential for $\mathrm{THz}$ emission. This dual-mode laser relies on the control of an initial Fabry-Pérot free spectral range by an etched photonic band gap [1]. In the indirect scheme presented here, the dual mode FP laser is used in the same experimental conditions to generate a $\mathrm{THz}$ signal with a photomixer composed of a UTC-PD monolithically integrated [5] with a transverse electromagnetic horn antenna (TEM-HA). The linewidth determination of the $\mathrm{THz}$ emission is often limited by the resolution of detection setups. In this paper, we experimentally investigate the linewidth of the $\mathrm{THz}$ signal generated using a room temperature electronic sub-harmonic mixer which offers the resolution of heterodyne electronic detection systems.

\section{EXPERIMENTAL SET-UP}

The experimental scheme is presented in Fig.1. The dual-mode laser is coupled to an optical fiber and amplified by an erbium doped fiber amplifier (EDFA). A wavemeter is used to monitor the optical spectrum emitted by the FP laser. The whole experimental setup was placed on an antivibrational workbench and the measurements were carried out at room temperature.

The laser is a $350 \mu \mathrm{m}$ long InAlGaAs multi-quantum well based FP device, mounted in a standard TO can. This device was previously described in [1,6]. Its $2 \mu \mathrm{m}$ wide ridge waveguide secures a single mode spatial output. It is characterized by a threshold current of $\mathrm{I}_{\mathrm{th}}=19 \mathrm{~mA}$. The fig. 2 presents the optical spectrum produced by the laser. The optical mode suppression ratio between the two main modes (1553 and and $1556 \mathrm{~nm}$ ) and the side-bands is greater than $30 \mathrm{~dB}$, so their direct contribution to the beat signal is negligible. This dual-wavelength signal is focused in the photomixer and $\mathrm{THz}$ signal is radiated in free space by the TEM-HA.

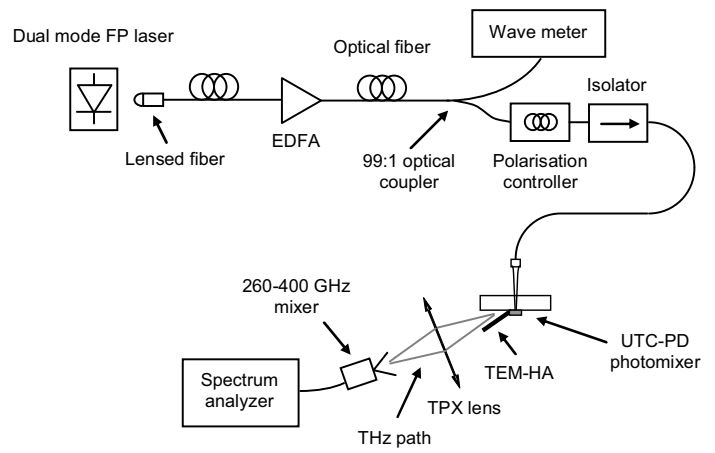

Fig. 1: Experimental setup

A polymethylpentene (TPX) based lens $(25 \mathrm{~mm}$ focal length) is used, in order to collect and focus the $\mathrm{THz}$ radiation, into a WR-2.8 (260-400 GHz) horn of an active sub harmonic mixer from RPG GmbH. This mixer is driven by a $7-15.5 \mathrm{GHz}$ local oscillator followed by an active multiplier. The intermediate frequency signal at $404.4 \mathrm{MHz}$ is resolved with a Rohde \& Schwarz FSU 67 spectrum analyzer. A $1 \mathrm{MHz}$ resolution bandwidth and a $20 \mathrm{~ms}$ sweep time were used, with no averaging.

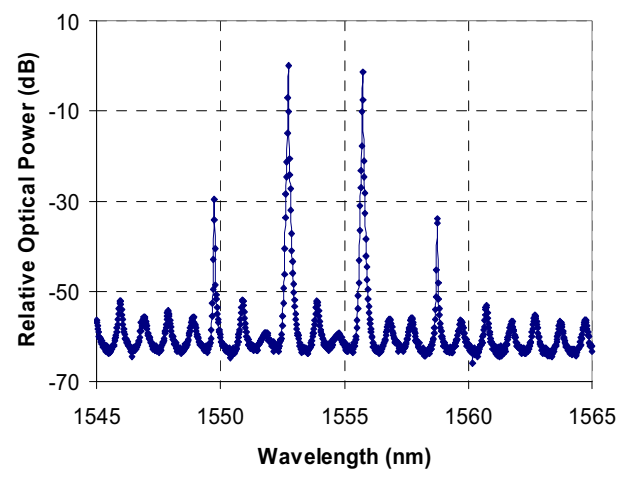

Fig. 2: Optical spectrum of the dual-mode FP laser for a 87.5 $\mathrm{mA}$ bias current.

\section{RESULTS}

The THz signal spectrum is shown in Fig.3. This spectrum has been fitted with a Lorentzian shape, exhibiting a central frequency at $371.895 \mathrm{GHz}$, corresponding to the frequency difference between the two main optical modes. Between 84 
and $91 \mathrm{~mA}$ of the injected bias current, the linewidth was experimentally measured between $17 \mathrm{MHz}$ and $40 \mathrm{MHz}$ while a $1200 \mathrm{MHz}$ tuning range was achieved as indicated by Fig.4. A quasi linear dependence with positive slope of central frequency is clearly observed.

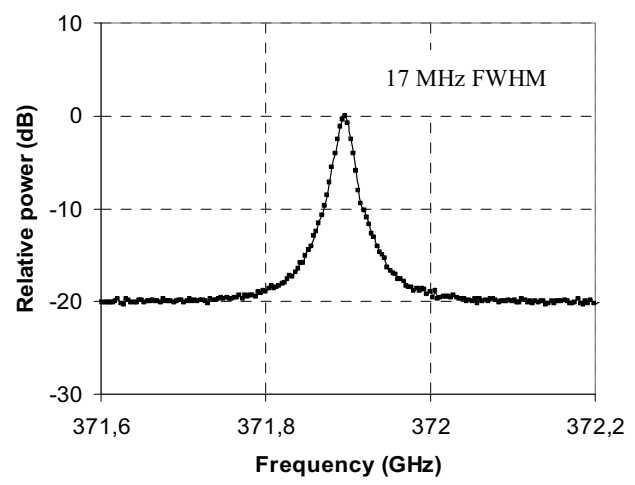

Fig. 3: Electrical detected spectrum at the spectrum analyzer corresponding to the optical spectrum photodetected by the UTC-PD and radiated in free space.

This behavior can be linked to the variation in the group refractive index of the active layer. As the bias current increases, the carrier density in the active layer increases. By plasma effect the group refractive index is reduced. The free spectral range increases and therefore the beat frequency also increases.

The measured $\mathrm{THz}$ peak power for a range of bias between 84 and $91 \mathrm{~mA}$ is also presented in the Fig. 4. Outside this current range, it is not possible to detect and to resolve the $\mathrm{THz}$ signal. The reason is that outside this current range, the optical spectrum from the FP laser presents a single mode emission and therefore no beat note is generated by UTC-PD. Last, the power is expressed in arbitrary unit as it was difficult to evaluate the losses due to the free space coupling.

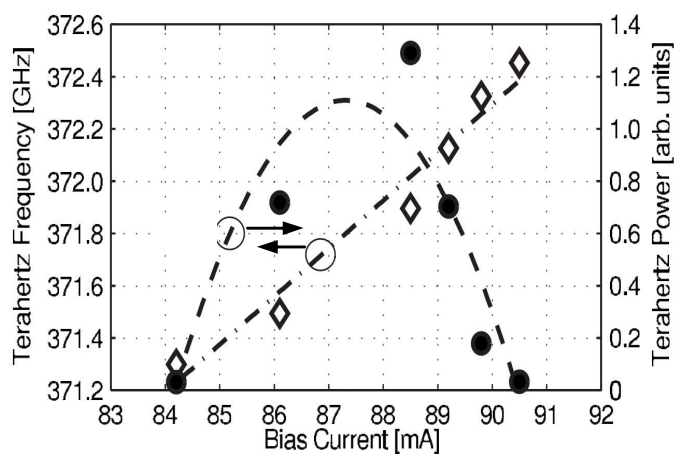

Fig. 4: Power of THz signal generated (O) and its central frequency $(\diamond)$ as a function of bias current applied to the laser diode. The dashed lines are eye-guides.

The THz signal linewidth has also been extracted as function of the bias current and the power unbalance (PU) between the two dominant optical modes for the same bias range are plotted in Fig. 5. Outside the range of bias current between 84 and 91 $\mathrm{mA}$, where the $\mathrm{THz}$ emission occurs, the PU is larger than 20 dB. At $83.5 \mathrm{~mA}$ the dominant mode is at $1553 \mathrm{~nm}$ while at 92
$\mathrm{mA}$, it is at $1556 \mathrm{~nm}$. The spectral purity of the $\mathrm{THz}$ signal appears to be linked to the PU. In the investigated bias range both quantities seem to follow a similar trend, achieving minimal values at the same bias current.

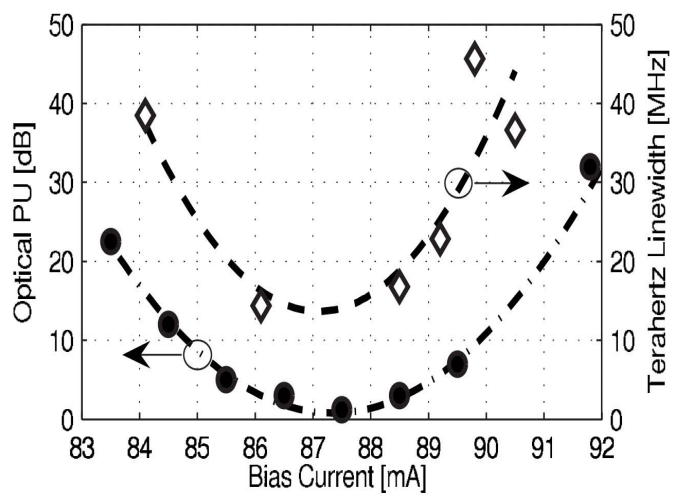

Fig. 5: Optical power unbalance $(\bigcirc)$ and THz linewidth $(\diamond)$ as a function of bias current applied to the laser diode. The dashed lines are eye-guides.

\section{CONCLUSION}

A room temperature generation of a narrowband $\mathrm{THz}$ signal based on a spectrum tailored semiconductor Fabry-Pérot laser and a UTC-PD has been demonstrated. The key feature of this scheme lies in the stability and the quality of the $\mathrm{THz}$ signal achieved.

For applications compatible with megahertz linewidths, semiconductor lasers are compact and cost effective. It should be noted that if the optical coupling between the laser and the fiber is enhanced, the EDFA could be removed from the setup. In this case a very compact, light-weight, and possibly battery operated $\mathrm{THz}$ source can be achieved.

\section{REFERENCES}

[1] S. Latkowski, F. Surre, and P. Landais, Applied Physics Letters 92, 081109 (Feb. 2008).

[2] S. Hoffmann, M. R. Hofmann, M. Osinski, H. Amano, and F. Henneberger, in Physics and Simulation of Optoelectronic Devices XII, Vol. 5349 (SPIE, San Jose, CA, USA, 2004) pp. 218-227.

[3] M. Hyodo, M. Tani, S. Matsuura, N. Onodera, and K. Sakai, Electron. Lett. 32, 1589 (1996).

[4] M. Tani, P. Gu, M. Hyodo, K. Sakai, and T. Hidaka, Optical and Quantum Electronics 32, 503 (2000).

[5] E. Peytavit, J.-F. Lampin, T. Akalin, and L. Desplanque, Electronics Letters 43, 73 (2007).

[6] R. Maldonado-Basilio, S. Latkowski, F. Surre, and P. Landais, Optics Communications 283, 299 (2010). 